\title{
Pharmacophore Modelling of Brassicaceae Members as Potent HIF (Hypoxia Inducible Factor) Inhibitors Involved in Cancer Angiogenesis
}

\author{
Jeyavel Renukadevi ${ }^{* 1}$, Ganesan Nandhinidevi ${ }^{1}$, Muthiah Bavanilatha ${ }^{2}$, Hemanath Tharani ${ }^{1}$, Rajarajan \\ Sathiyabama', Subramani Vasumathi'
}

\section{Jeyavel Renukadevi*1, Ganesan Nandhinidevi', Muthiah Bavanilatha ${ }^{2}$, Hemanath Tharani' ${ }^{1}$, Rajarajan Sathiyabama ${ }^{1}$, Subramani Vasumathi ${ }^{1}$}

'Department of Biotechnology, Anna University, Chennai, Tamil Nadu, INDIA. 2Department of Biotechnology, Sathyabama University, Chennai, Tamil Nadu, INDIA.

Correspondence

Jeyavel Renukadevi

Department of Biotechnology, Anna University, Chennai-600025, Tamil Nadu, INDIA.

E-mail: neelarenu@gmail.com

History

- Submission Date: 27-10-2017;

- Review completed: 05-03-2018;

- Accepted Date: 12-03-2018

DOI : 10.5530/pj.2018.4.135

Article Available online

http://www.phcogj.com/v10/i4

\section{Copyright}

(C) 2018 Phcog.Net. This is an openaccess article distributed under the terms of the Creative Commons Attribution 4.0 International license.

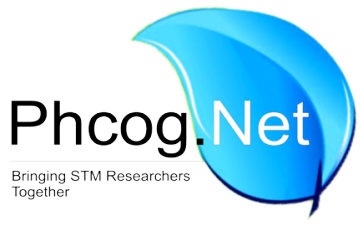

\section{ABSTRACT}

Angiogenesis is considered as an essential pathological feature of cancer due to its interplay between cancer and other diseases. Natural products found to act as antiangiogenic agents that mediate the angiogenic switch between pro and anti angiogenic factors. Among the different targets, HIF is an important and critical factor that stands as a key mediator between angiogenesis, inflammation and cancer. In our study different phytochemicals of Brassicaceae were analysed for their drug like properties and mapped for pharmacophore development. The developed pharmacophore was virtually screened and further subjected to Lipinski and ADMET filters. The molecular interaction studies of the 10 retrieved compounds were studied by binding with HIF. Among the compounds $1^{\text {st }}$ drug like molecule HTS $0115\left(\mathrm{C}_{15} \mathrm{H}_{21} \mathrm{BrN}_{2} \mathrm{O}_{3}\right)$ was found to have best docked score and its interaction was further validated using dynamics simulation. The compound found to share the pharmacophoric features with progoitrin a biochemical form of glucosinolate with reported anticancer and anti thyroid activities. Thus the drug like compound HTS 0115 can be further optimised as a putative HIF inhibitor in tumor angiogenesis.

Key words: Pharmacophore, HIF, Angiogenesis, Brassicaceae, Molecular docking, Simulation.

\section{INTRODUCTION}

Angiogenesis is a physiological process involved in blood vessel formation related to several developmental process in embryo,placenta and wound healing. It is controlled by a tenuous balance between angiogenic and angiostatic factors. ${ }^{1}$ In cancer, this imbalance is getting activated in the endothelial cell leading to metastatic proliferation. When a small dormant tumor initiates angiogenesis, referred to as the 'angiogenic switch', it secretes factors that induce sprouting and chemotaxis of endothelial cells (ECs) towards the tumor mass. ${ }^{2}$ Within this hypoxic environment the transcription factor Hypoxia-Inducible-Factor-1- (HIF-1) is stabilized that activates the expression of multiple genes contributing to the angiogenic process. HIF-1 induced proteins include Vascular Endothelial Growth 2 Factor (VEGF) and Basic Fibroblast Growth Factor (bFGF), which promote vascular permeability and growth, respectively. Thus HIF expression is considered to be the key signalling factor in cancer angiogenesis. Other HIF- 1 induced gene products include matrix metalloproteinases (MMPs) that breakdown the extracellular matrix to facilitate extracellular migration release growth factors such as integrins and tumor-associated macrophages (TAMs). ${ }^{3}$ Cruciferous vegetables were extensively studied for their anticancer properties due o the presence of glucosinolates and sulforaphane. ${ }^{4} \mathrm{Glu}$ cosinolates are hydrolyzed to isothiocyanates (ITCs). (ITCs), a bioactive component in cruciferous vegetables that are being investigated for their chemopreventive and chemotherapeutic effects. These chemicals are responsible for the pungent aroma and bitter flavour of cruciferous vegetables. Indole-3- carbinol (an indole) and sulforaphane (an isothiocyanate) are the most frequently examined phytochemicals for their anticancer effects. ${ }^{6}$ The purpose of the study is to design a pharmacophore model based on the interaction of different phytoconstituents of Brassicaceae with HIF and optimising it as a lead against angiogenesis. The model was developed using common pharmacophore and ligand mapping followed by its validation using Molecular Dynamics (MD) simulation.

\section{MATERIALS AND METHODS}

The study is designed for pharmacophore candidate from set of potent ligands with reported activities. Pharmacophore features were devised from set of ligands using PHARMAGIST an online web server

Citethisarticle: RenukadeviJ, Nandhinidevi G, BavanilathaM,TharaniH, SathiyabhamaR,VasumathiS Pharmacophore Modelling of Brassicaceae Members as Potent HIF (Hypoxia Inducible Factor) Inhibitors Involved in Cancer Angiogenesis. Pharmacog J. 2018;10(4):798-802. 
It involves an indirect approach which requires a $3 \mathrm{D}$ training set that serves as a pivot point for alignment. The input set include the phytochemicals that are known to bind to HIF. The output consists of candidate pharmacophores that are generated by MFA (molecular field analysis).It employs the flexibility of input ligands in a deterministic manner within the alignment process.

\section{Identification and screening of phytoconstituents and their studies}

Anticancer and antiangiogenic compounds from different members of Brassicaceae were collected and reviewed. From the collection the compounds with reported anti angiogenic properties were separated and thoroughly studied using wide literature collection. From the list, constituents were screened for drug like properties using Lipinski rule of five. The resultant ligands were devised for their pharmacophore features using PHARMAGIST.

\section{Pharmacophore model development and virtual screening}

The compounds with potent antiangiogenic properties were used as base template for the generation of pharmacophore using COMMON PHARMACOPHORE GENERATION of Accelyrs Discovery Studio 2.0. (ADS). Different hypotheses were developed with different parameters such as $\mathrm{H}$-bond acceptor, donor and hydrophobic characteristics in common. The ligand pharmacophore and mapping protocol of ADS was used for mapping the best compounds with distinct pharmacophore features. Virtual screening of compounds was done with the developed pharmacophore in MINIMAY bridge database of ADS. The best pharmacophore models were chosen based on their structure likeness with the developed pharmacophore. All retrieved compounds were validated using drug likeness, ADMET descriptors and TOPKAT module of ADS. ${ }^{7,8}$

\section{Binding site analysis and Molecular docking}

The binding site was identified from receptor cavities using floodfilling algorithms as implemented in ADS. ${ }^{9}$ A grid resolution of 0.5 which is an indication of grid spacing and minimum number of grid points used for this analysis. ${ }^{10,11}$ The compounds with good drug likeness and ADMET properties were chosen and docked to the binding site of HIF using AUTODOCK of ADS 2.0. The resulting docked complexes were selected based on dock score and PMF (potential of mean force).

\section{Molecular dynamics simulation}

Molecular dynamics simulation is a computational method that calculates the time-dependent behaviour of a molecular system. Molecular dynamics simulations provide detailed information on the fluctuations and conformational changes of proteins and nucleic acids. These methods are used

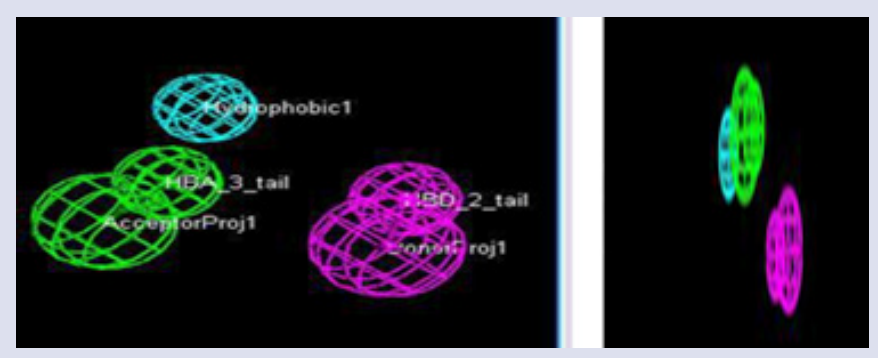

Figure 1: (a)Common pharmacophore 1(b) Ligand mapping. to investigate the structure, and thermodynamics of biological molecules and their complexes. The complex of drug like compound with target protein HIF was analyzed by producing simulation at $200 \mathrm{~K}$ for 1000 ns of the complex. The dynamics was then analyzed and the interacting residues were found. ${ }^{12,13}$

\section{RESULTS AND DISCUSSION}

\section{Target protein preparation}

The $3 \mathrm{D}$ structure of HIF was retrieved from PDB and stabilised for docking. The structure was minimised with conjugate gradient to resolve steric clashes and unwanted interactions.

\section{Identification and screening of phytoconstituents and their studies}

From the literature search different ligands from Brassicaceae were selected.29 ligands were screened using Lipinski filter and the structures retrieved from PUBCHEM were used for the analysis. The list of ligands used for pharmacophore mapping were shown in Table 1.

\section{Pharmacophore model generation}

The compounds with good drug likeness were used as base structures for the generation of pharmacophore using PHARMAGIST. The pharmacophoric features were analysed for the 19 ligands using PHARMAGIST. Pharmacophore model generation was done using COMMON FEATURE PHARMACOPHORE TOOL IN ADS. ${ }^{14}$ Four ligands such as Kaempferol, Glucoraphanin, Flavanol, Progoitrin were found to have best pairwise alignment with flavanol as the pivotal molecule. Ligands such as Progoitrin and Kaempferol were found to be best mapped with generated pharmacophore. Drug likeness of the above two compounds were further screened for their analogues using database. Compounds with best fit values were subjected to toxicity prediction and pharmacokinetics using ADS as shown in Figure 1(a) and (b).

HIF was found to have 10 binding sites and the docking was done using ligand fit module by Monte Carlo methods. ${ }^{15}$ The final docked poses were evaluated based on dock scores and hydrogen bonding interaction with the binding site residues as shown in Table 2 and Figure 2. ${ }^{15}$

\section{Molecular Dynamics simulation}

Molecular dynamics (MD) is a method of studying the motions in the conformational space of molecular systems. MD simulation was performed over period of $1000 \mathrm{~ns}$ with the temperature at $200 \mathrm{~K}$ for all selected top ten drug molecules with HIF. The model produced after simulation shows that the HIS115, SER 11 and TYR 98 of HIF interact with HIS 56, ALA 3 and TYR 5 of the $1^{\text {st }}$ drug like compound. The $10 \mathrm{~mol}$ of compound showed the interaction of ALA 3 and LEU 2. The binding complex of $1^{\text {st }}$ mole with HIF complex showed stable conformation throughout the simulation process. The drug like compound HTS $0115(\mathrm{C} 15 \mathrm{H} 21 \mathrm{BrN} 2 \mathrm{O} 3)$ found to have the highest dock score with the receptor as shown in Figure 3. It has the same pharmacophoric features as PROGOITRIN.

Progoitrin is a biochemical form of the glucosinolate ( 2 hydroxy 3 butenyl) derivative predominantly found in cabbage, broccoli, brussel sprouts and other members of Brassicaceae. ${ }^{16}$ It is reported to possess anticancer and antiangiogenic properties. It is hydrolysed by myrosinase to form cyclic thiocarbamate goitrin which is found to reduce the production of thyroid hormone. There exists an inverse relationship between the thyroid vasculature and the local availability of iodine mediated by trace element dependant regulation of VEGF (vascular endothelial growth factor). HIF is responsible for VEGF activation by perceiving decreased iodide concentration as pseudo hypoxic stimulus. ${ }^{17,18}$ Therefore, we conclude 


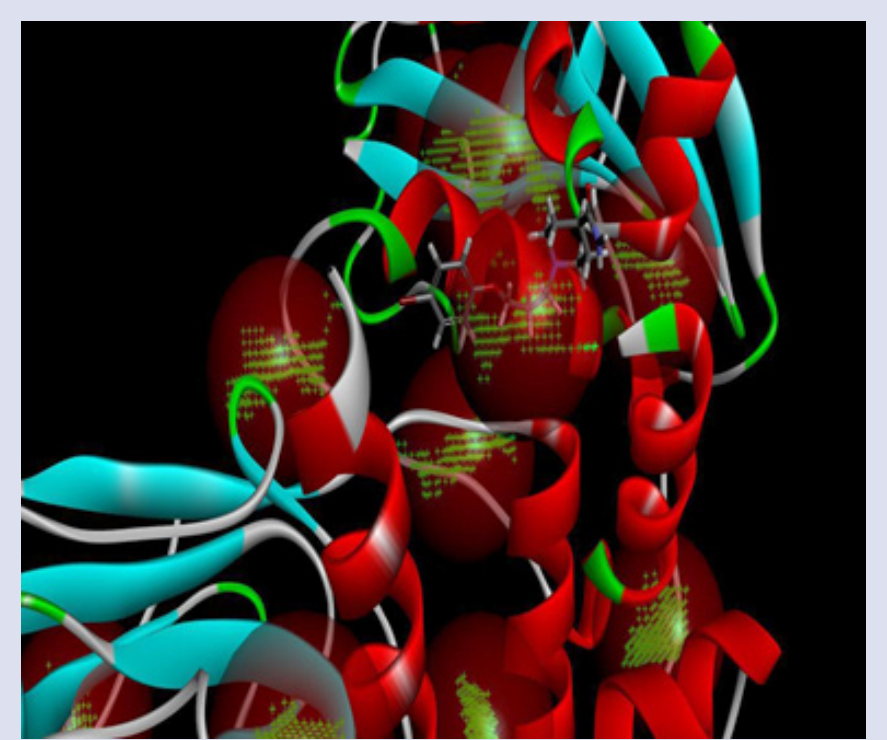

Figure 2: Molecular docking of HIF with developed pharmacophore (HTS 0115 molecule) .

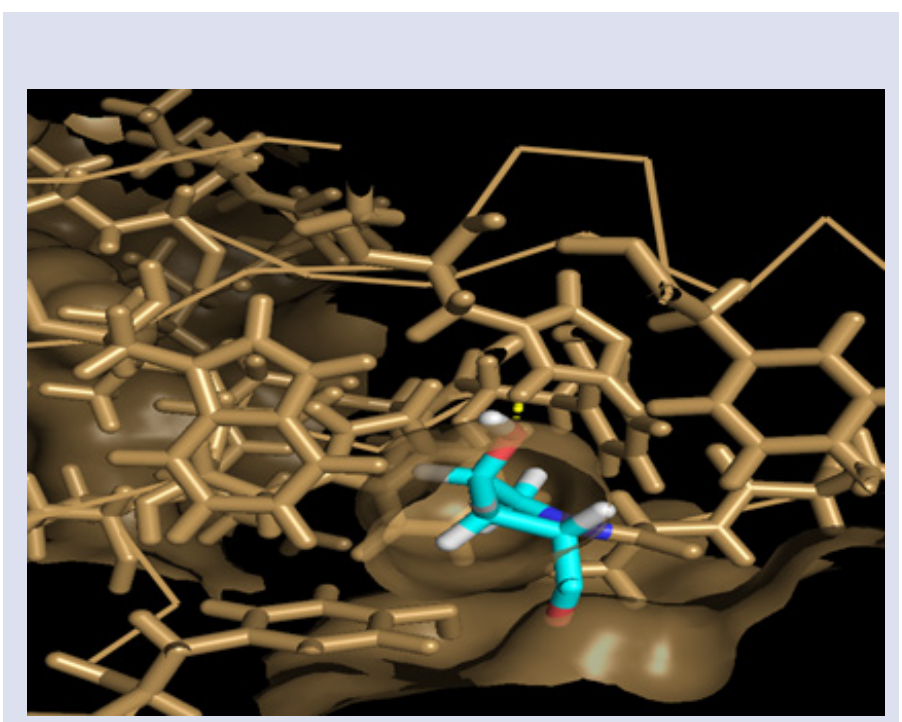

Figure 3: Molecular dynamics simulation of HIF with HTS 0115 molecule.

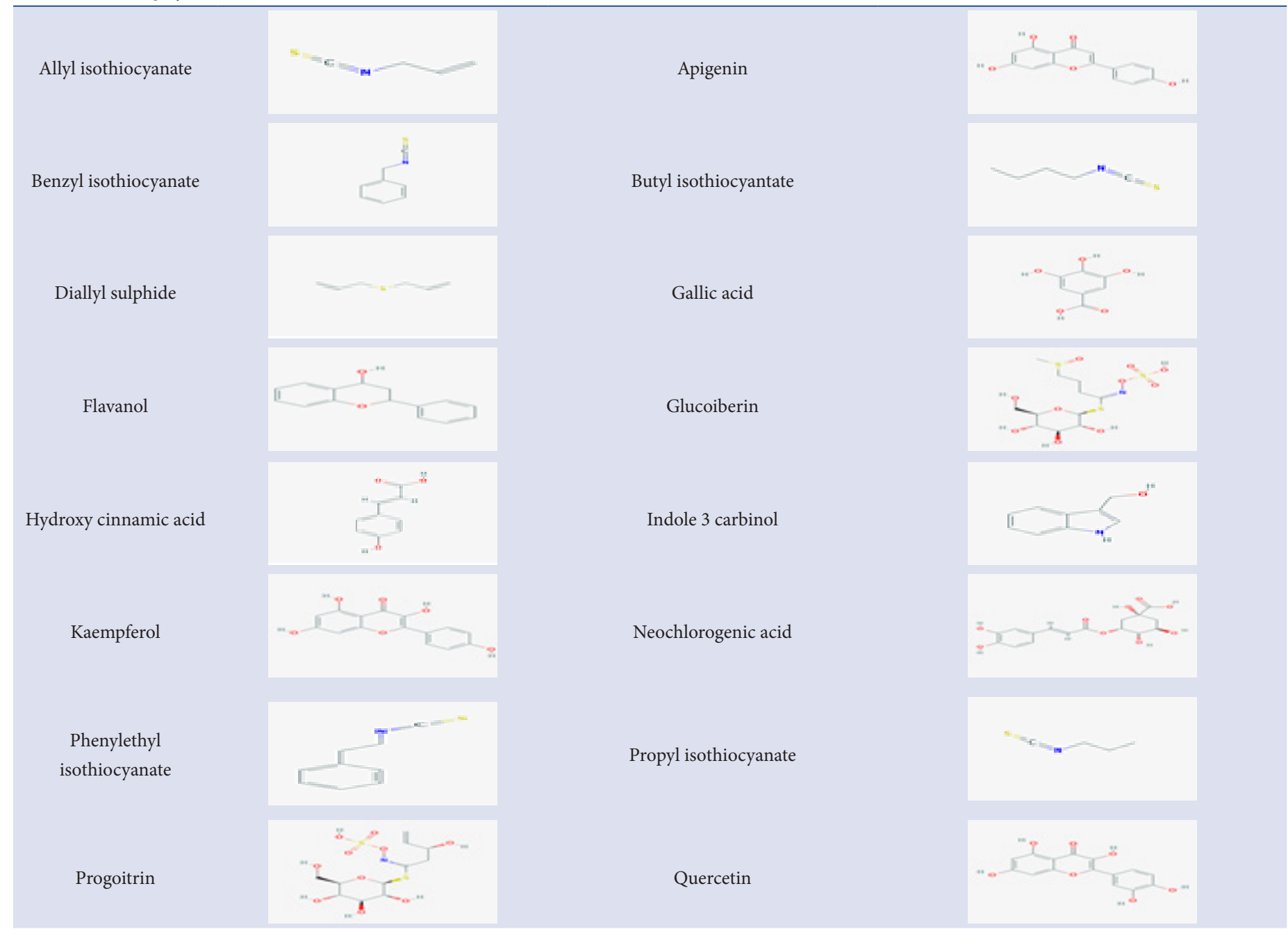


Table 2: Docked result of 10 molecules with HIF receptor

\begin{tabular}{cccc}
\hline Site number & Molecule & PMF & Dock score \\
\hline 1 & HTS 01115 & 69.26 & 21.26 \\
2 & RDR 03478 & -1.15 & 26.17 \\
3 & JFD 03560 & 23.9 & 34.47 \\
4 & BTB 02602 & -38.65 & 21.64 \\
5 & KM 06492 & 20.34 & 32.68 \\
6 & S 01184 & 32.04 & 47.27 \\
7 & JFD 03350 & 0 & 0 \\
8 & JFD 03564 & 0 & 0 \\
9 & BTB 14837 & 0 & 0 \\
10 & RDR 01650 & 0 & 0 \\
\hline
\end{tabular}

that molecule HTS 0115 (C15H21BrN2O3) has ANTI ANGIOGENIC property and further be optimised as an angiogenic inhibitor using in vitro and in vivo studies.

\section{CONCLUSION}

Natural product research recently focuses on optimising the phytoconstituents as druggable leads in different signalling mechanism associated with cancer. Targeting intervening mechanism such as angiogenesis associated with cancer may repurpose existing leads for new therapeutic indications. In our study we devised pharmacophoe mapping from Brassicaceae and their molecular interaction to explore suitable HIF inhibitors. Among the drug like compounds reported from database, HTS 0115 (C15H21BrN2O3) with best dock score was validated using simulation studies. It shares the same pharmacophoric features of Progoitrin a biochemical form of glucosinolate. Hence it may be optimised in future as an effective natural lead in tumor angiogenesis.

\section{CONFLICT OF INTEREST}

The authors declare no conflict of interest.

\section{ABBREVIATIONS}

ADS: Accelyrs discovery Studio; ITC: isothiocyanates; PMF: potential mean force; HIF: Hypoxia inducible factor; ALA: Alanine; THR: Threonine; TRP: Tryptophan.

\section{REFERENCES}

1. Wang Z, Dabrosin C, Yin X, Fuster M, Arreola A, Rathmell W et al. Broad targeting of angiogenesis for cancer prevention and therapy. Semin Cancer Biol. 2015;35:S224-43.

2. Lakka SS, Rao JS. Angiogenic therapy in brain tumors. Expert Rev Neurother. 2008:8(10):1457-73

3. Kessenbrock K, Placks V, Werb Z. Matrix metalloproteinases: Regulators of the tumor microenvironment. Cell. 2010:141(1):52-67.

4. Ishida M, Hara M, Fukino N, Kakizaki T, Morimitsu Y. Glucosinolate metabolism, functionality and breeding for the improvement of Brassicaceae vegetables. Breed Sci. 2014;64(1):48-59.

5. Laing B, Han D, Ferguson L. Candidate Genes Involved in Beneficial or Adverse Responses to Commonly Eaten Brassica Vegetables in a New Zealand Crohn's Disease Cohort. Nutrients. 2013;5(12):5046-64.

6. Novío S, Cartea M, Soengas P, Freire-Garabal M, Nunez-Iglesias M. Effects of Brassicaceae Isothiocyanates on Prostate Cancer. Molecules. 2016;21(5):626.

7. Agrawal R, Jain P, Narayan Dikshit S. Ligand-Based Pharmacophore Detection, Screening of Potential Gliptins and Docking Studies to Get Effective Antidiabetic Agents. Comb Chem High throughput Screen. 2012;15(10):849-76.

8. Kumar R, Suresh M. Pharmacophore mapping based inhibitor selection and molecular interaction studies for identification of potential drugs on calcium activated potassium channel blockers, tamulotoxin. Pharmacogn Mag. 2013; 9(34):89.

9. Venkatachalam C, Jiang X, Oldfield T, Waldman M. Ligand Fit: A novel method for the shape-directed rapid docking of ligands to protein active sites. J Mol Graph Model. 2003;21(4):289-307.

10. Fei J, Zhou L, Liu T, Tang X. Pharmacophore Modelling, Virtual Screening, and Molecular Docking Studies for Discovery of Novel Akt2 Inhibitors. Int J Med Sci. 2013;10(3):265-75.

11. Kavitha R, Karunagaran S, Chandrabose S, Lee K, Meganathan C. Pharmacophore modelling, virtual screening, molecular docking studies and density functional theory approaches to identify novel ketohexokinase (KHK) inhibitors. Biosystems. 2015;138:39-52.

12. Meng X, Zhang H, Mezei M, Cui M. Molecular Docking: A Powerful Approach for Structure-Based Drug Discovery. Curr Comput-Aided Drug Des. 2011;7(2):146-57.

13. Hospital A, Goni R, Orozco M, Gelpi J. Molecular dynamics simulations: advances and applications. Adv Appl Bioinform and Chem. 2015;8:37-47.

14. Levit A, Yarnitzky T, Wiener A, Meidan R, Niv M. Modeling of Human Prokineticin Receptors: Interactions with Novel Small-Molecule Binders and Potentia Off-Target Drugs. PLoS ONE. 2011;6(11):e2799.

15. Srivastava V, Kumar A, Mishra BN, Siddiqi MI. Molecular docking studies on DMDP derivatives as human DHFR inhibitors. Bioinformation. 2008;3(4):180-8

16. Kushad MM, Brown CA, Kurilich CA, Juvik JA, Klein BP, Wallig MA, Jeffrey EH. Variation of glucosinolates in vegetable crops of Brassica oleraceae. J Agric Food Chem.1999;49(4):1541-8.

17. Gerard A, Poncin S, Audinot J, Denef J, Colin I. lodide deficiency-induced angiogenic stimulus in the thyroid occurs via HIF- and ROS-dependent VEGF-A secretion from thyrocytes. AJP: Endocrinol and Metabs. 2009;296(6):E1414-22.

18. Gerard A, Humblet K, Wilvers $\mathrm{C}$, Poncin S, Derradji $\mathrm{H}$, de Ville de Goyet $\mathrm{C}$ et al. lodine deficiency-induced long lasting angiogenic reaction in thyroid cancers occurs via a VEGF-HIF-1, but not a ROS dependent pathway. Thyroid. 2012; 22(7):699-708. 120403103408002.

Cite this article: Renukadevi J, Nandhinidevi G, Bavanilatha M, Tharani H, Sathiyabhama R, Vasumathi S. Pharmacophore Modelling of Brassicaceae Members as Potent HIF (Hypoxia Inducible Factor) Inhibitors Involved in Cancer Angiogenesis. Pharmacog J. 2018;10(4):798-802. 
GRAPHICAL ABSTRACT

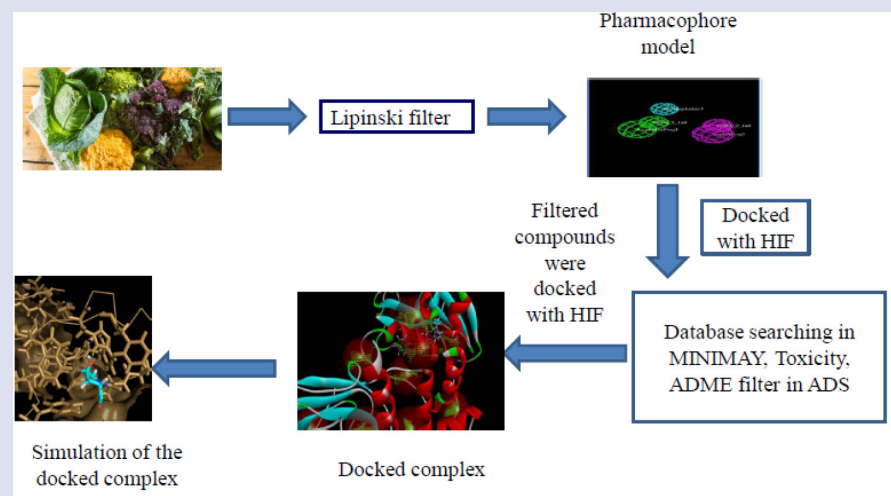

\section{SUMMARY}

- A pharmacophore model was built using phytochemicals of Brassicaceae family as an effective HIF (hypoxia inducible factor) inhibitor using pharmacophore based ligand mapping The model was used as a key molecule and compared with compounds in database, similar compounds were filtered and docked with the receptor. Among the different compounds, the 1st molecule which shares the pharmacophoric features of Progoitrin was bound with the HIF receptor. The docked complex was found to stable throughout the entire simulation and annealing process. 